\title{
Numerical modeling of the filament-assisted diamond growth environment
}

\author{
D. G. Goodwin and G. G. Gavillet \\ Division of Engineering and Applied Science, California Institute of Technology, Pasadena. \\ California 91125
}

(Received 8 May 1990; accepted for publication 27 August 1990)

\begin{abstract}
A numerical model of the filament-assisted diamond growth environment has been developed and used to calculate temperature, velocity, and species concentration profiles, accounting both for transport and detailed chemical kinetics. The computed hydrocarbon concentrations agree well with previously measured values, when allowance is made for 3D effects not included in our model. Upper-bound, diffusion-limited film growth rates for various assumed growth species have been computed, and it has been found no hydrocarbon species other than $\mathrm{CH}_{3}, \mathrm{C}_{2} \mathrm{H}_{2}$, or $\mathrm{CH}_{4}$ can account for measured diamond growth rates. The effect of thermal diffusion on $\mathrm{H}$-atom profiles has been examined, and found to be only $10 \%$. Although the environment is far from thermodynamic equilibrium, several reactions are close to partial equilibrium throughout the region from the filament to the substrate. It is also shown that homogeneous $\mathbf{H}$-atom recombination is too slow to explain the experimentally observed decrease in the concentration of $\mathrm{H}$ with increasing initial methane concentration.
\end{abstract}

\section{INTRODUCTION}

Diamond is currently grown using many different techniques, ranging from low-pressure plasma and thermal processes to high-speed dc arcjets to oxyacetylene torches. ${ }^{1}$ Studies of the gas-phase environment in which diamond nucleation and growth occurs are of considerable interest, since a knowledge of the gas composition at the substrate may give important clues regarding the kinetic mechanism leading to diamond formation. In addition, variations in gas composition may at least partly explain the empirically observed composition and temperature range over which crystalline diamond may be grown, as well as the dependence of film quality on process conditions.

At present, quantitative species concentrations have been reported only for the filament-assisted environment. ${ }^{2-7}$ Since this is a purely thermal process, the gas-phase chemistry is also easier to model and understand for this system than it is for plasma processes. Of the many gaseous diamond precursors which have been proposed, in situ concentration measurements in filament-assisted reactors, combined with kinetic arguments, have identified the most likely species as $\mathrm{CH}_{3}, \mathrm{C}_{2} \mathrm{H}_{2}, \mathrm{C}_{2} \mathrm{H}_{4}$, and $\mathrm{CH}_{4}{ }^{2,5}$ Reactivity considerations ${ }^{5}$ suggest that of these, either the methyl radical or acetylene is the probable growth species.

Theoretical mechanisms have been presented both for diamond growth from acetylene ${ }^{8}$ and for growth from methyl. ${ }^{9,10}$ Molecular dynamics calculations ${ }^{11}$ indicate that the original methyl mechanism of Tsuda and co-workers ${ }^{9}$ is unlikely, since it appears to have a large activation energy ( 210 $\mathrm{kcal} / \mathrm{mol}$ ). However, Harris ${ }^{10}$ has recently proposed a mechanism for growth from $\mathrm{CH}_{3}$ on the (100) surface of diamond, and has shown that the mechanism is feasible, to the extent that each step has $\Delta G<0$ and predicted growth rates are of the same order as those measured. Recent experimental evidence obtained in a low-pressure flowtube reactor appears to point to a $\mathrm{C}_{1}$ hydrocarbon as the precursor to diamond. ${ }^{12}$
Detailed numerical modeling of the gaseous environment can serve as a useful complement to experimental studies, providing spatially resolved velocity, temperature, and concentration fields that may be compared to experiment. Carefully validated models can provide significant insights which are difficult to obtain from experiment alone. The value of detailed modeling has been convincingly demonstrated in other chemical vapor deposition systems, such as silicon deposition, for which temperature and species $\left(\mathrm{SiH}_{4}\right.$, $\mathrm{Si}, \mathrm{Si}_{2}$ ) profiles have been calculated which agree very well with the those measured using laser probes. ${ }^{13}$

For studies of diamond growth, the only detailed modeling presented to date has been for atmospheric pressure thermal plasmas. ${ }^{14}$ For the filament-assisted environment, only zero-dimensional kinetic calculations have been presented, using an assumed temperature history, though in some cases diffusion has been included approximately. ${ }^{5.7 .15 .16}$

A satisfactory model of the gaseous environment requires solving for the velocity, temperature, and concentration fields, including both convective and diffusive transport, together with a detailed reaction mechanism. To model the actual reactor geometries currently in use would require at least a two-dimensional model, and in many cases, a threedimensional one, which becomes computationally prohibitive if detailed chemistry is included.

We have chosen instead to model a simplified geometry which retains the essential physical characteristics of a real reactor, but allows a one-dimensional formulation of the governing equations. In this way, we can calculate exact velocity, temperature, and species concentration profiles near the substrate, even though we do not model precisely the geometry of current diamond growth reactors. For future experimental work, a reactor could easily be designed which would approximate the geometry we assume here.

The model is formulated generally, and may be used to investigate both high- and low-pressure diamond synthesis environments. In the present paper, we restrict our attention to the filament-assisted environment, and compare our re- 
sults with the available experimental data for species concentrations and film growth rates.

\section{MODEL}

Our approach follows closely that employed by Coltrin $\mathrm{Kee}$, and Evans in their study of silicon CVD in a rotating disk reactor. ${ }^{17}$ We consider a porous, infinite-radius plate (the gas inlet) located at $z=L$, through which gas is forced, which emerges with a uniform, purely axial downward gas velocity $-u_{L}$. The substrate, also of infinite radius, is located at $z=0$. We seek the solution for the velocity, temperature, and species concentration fields in the gap between the inlet and the substrate, $0 \leqslant z \leqslant L, 0 \leqslant r \leqslant \infty$. For complete generality, we allow the substrate to rotate with angular velocity $\Omega$, although we are interested here only in the case $\Omega=0$.

The flowfield is determined by solving the conservation equations for mass, momentum, energy, and species with appropriate boundary conditions at the inlet and substrate. It may be shown ${ }^{17}$ that the conservation equations admit an axisymmetric similarity solution for this geometry, with the properties that the axial, radial, and circumferential velocity components $u, v, u$ are given by $u=u(z), v=r V(z)$, and $u=r W(z)$, respectively. Furthermore, the temperature $T(z)$ and species mass fractions $Y_{k}(z)$ are independent of $r$, and the pressure has the form

$$
p(r, z)=p_{0}(z)+\Lambda r^{2} / 2,
$$

where $A$ is constant.

Substituting these forms into the exact, three-dimensional conservation equations results in the following set of ordinary differential equations in $z$.

Continuity:

$$
\frac{d u}{d z}+2 V+\frac{u}{\rho} \frac{d \rho}{d z}=0
$$

radial momentum:

$\rho u \frac{d V}{d z}+\rho\left(V^{2}-W^{2}\right)-\frac{d}{d z}\left(\mu \frac{d V}{d z}\right)+\Lambda=0 ;$

axial momentum:

$\rho u \frac{d u}{d z}-2 \mu \frac{d V}{d z}-\frac{4 d}{3} \frac{d}{d z}\left[\mu\left(\frac{d u}{d z}-V\right)\right]+\frac{d p}{d z}=0$

circumferential momentum

$$
\rho u \frac{d W}{d z}+2 \rho V W-\frac{d}{d z}\left(\mu \frac{d W}{d z}\right)=0
$$

energy:

$\rho c_{p} u \frac{d T}{d z}$

$$
+\sum_{\grave{h}}\left(\rho c_{p, h} U_{k} Y_{k} \frac{d T}{d z}+M_{k} \dot{\omega}_{k} h_{k}\right)-\frac{d}{d z}\left(\lambda \frac{d T}{d z}\right)=0 ;
$$

species conservation:

$\rho u \frac{d Y_{h}}{d z}+\frac{d}{d z}\left(\rho U_{k} Y_{k}\right)-\dot{\omega}_{k} M_{k}=0 ;$

equation of state:

$p_{0}=\rho R T$.
Using Eq. (8) as the equation of state instead of the exact form $p=\rho R T$ is necessary to obtain a set of equations that is independent of $r$. Consequently, a necessary condition for the similarity solution to hold is that $p_{0}(z) \gg \Lambda r^{2} / 2$.

Two further minor assumptions are required to yield this set of equations. Buoyancy effects must be negligible for the similarity solution to be valid. Although the buoyancy term - $\rho g z$ may be easily added to Eq. (4) without violating similarity, the entire similarity solution will be unstable with respect to development of convection cells if the density is unstably stratified $(d T / d z<0)$ and the Rayleigh number is greater than the critical value. However, for the flows we are interested in here, the density is stably stratified $(d T / d z>0)$ and the Rayleigh number is in any case far less than critical. Of course, for finite-radius geometries, buoyancy can be important. ${ }^{18}$

In deriving Eq. (6), we have also discarded the flowwork and viscous dissipation terms which appear in the general energy equation. ${ }^{19}$ These terms, which cannot be reduced to similarity form, are only important in high-velocity flows (typically Mach number $>1$ ). For the cases of interest here, the neglect of these terms is a very good approximation.

The term $U_{k}$ in Eqs. (6) and (7) is the diffusion velocity of species $k$. In the present version of our model, we use mixture-averaged mass diffusion coefficients, and include thermal diffusion only for $\mathrm{H}$ and $\mathrm{H}_{2}$. The diffusion velocity is assumed to be given by

$$
U_{k}=\frac{D_{k}}{X_{k}}\left[\frac{\Theta_{k}}{T} \frac{d T}{d z}-\frac{d X_{k}}{d z}\right]
$$

where $X_{k}$ is the mole fraction of species $k, D_{k}$ is the mixture diffusion coefficient, and $\Theta_{k}$ is the thermal diffusion ratio, taken to be nonzero only for $\mathrm{H}$ and $\mathrm{H}_{2}$.

We use the CHEMKIN-II subroutine package ${ }^{20}$ to facilitate the evaluation of the thermodynamic and chemical terms in our equations. The related transport property package $^{21}$ is used to evaluate the viscosity $\mu$, thermal conductivity $\lambda$, mixture diffusion coefficients $D_{k}$, and thermal diffusion ratios $\Theta_{k}$

The velocity boundary conditions are that $V(0)=V(L)=0, \quad W(0)=\Omega, \quad W(L)=0, \quad$ and $u(L)$ $=-u_{L}$. The axial velocity boundary condition at the substrate is that $\rho u(0)=-\dot{m}_{d}$, where $\dot{m}_{d}$ is the mass deposition rate $\left(\mathrm{g} / \mathrm{cm}^{2} / \mathrm{s}\right)$. For the hot-filament conditions we are interested in here, measured film growth rates imply that $u(0) \approx-0.015 \mathrm{~cm} / \mathrm{s}$. In light of this, we simply take the boundary condition $u(0)=0$.

The temperature is specified at the inlet and at the substrate: $T(L)=T_{i}, T(0)=T_{3}$. The species mass fractions $Y_{k}$ are assumed specified at the inlet. As will be discussed below, to model hot-filament diamond growth we choose $T_{i}$ and $Y_{k}$ at the inlet to be representative of gas which has passed near the filament. At the substrate, we assume that each carbon-bearing species has a probability $\gamma_{k}$ of reacting on the substrate surface, incorporating its carbon atoms into the diamond lattice and releasing its hydrogen in molecular form. (A better assumption would be that hydrogen leaves the surface as an equilibrium mixture of $\mathrm{H}$ and $\mathrm{H}_{2}$, but at a surface temperature of $1000 \mathrm{~K}$, the equilibrium atomic $\mathrm{H}$ 
mole fraction is negligible.) Consequently, for carbon-bearing species we apply the boundary condition

$$
\left[\rho Y_{k}\left(u+U_{k}\right)\right]_{z=0}=\frac{\gamma_{k}}{1-\left(\gamma_{k} / 2\right)} m_{k}\left(\frac{n_{k} \bar{c}_{k}}{4}\right),
$$

where $m_{k}$ is the mass of one $k$ molecule, $\bar{c}_{k}$ is the mean thermal speed ( $=\sqrt{8 k T / \pi m_{k}}$ ), and $n_{k}$ is the number density of species $k$. This expression differs by the factor $1-\gamma_{k} / 2$ in the denominator from the standard boundary condition (e.g., Ref. 22). For $\gamma_{k} \ll 1$, the two expressions agree, but for $\gamma_{k} \rightarrow 1$, Eq. (10) results in a surface concentration half that which would be predicted otherwise. It may be shown ${ }^{23,24}$ that Eq. (10) is the more correct expression, and accounts to first order for the non-Maxwellian velocity distribution of species $k$ near the surface when $\gamma_{k}$ is large. The boundary condition for $\mathrm{H}_{2}$ is that the flux of $\mathrm{H}_{2}$ away from the surface balances the hydrogen released at the surface in surface reactions.

We solve the full set of Eqs. (2-8), subject to these boundary conditions, to determine the flowfield. If we were to make the additional assumption that $p_{0}(z)$ is constant, it would be possible to avoid solving the axial momentum equation. For hot-filament conditions, this assumption is fully justified, as it is for the silicon CVD simulations of Ref. 17, where axial momentum was eliminated from the set of equations. However, we include axial momentum, since the added computational expense is small, and we may then calculate general cases with nonconstant $p_{0}(z)$. We find in addition that examining $p_{0}(z)$ is often a useful indicator of whether or not a solution is fully converged.

The set of equations is solved using a hybrid Newton/ time-integration scheme, based on the approach used by Grcar $e t a l^{25}$ for laminar, premixed flames and by Coltrin and co-workers ${ }^{17}$ for silicon CVD. The computation is divided in two major phases. First, an initial guess is made for the velocity and temperature profiles, chemistry is turned off, and an initial value of the parameter $\Lambda$ is chosen. The model converges quickly to a solution with a certain inlet axial velocity $u(L ; \Lambda)$, which depends parametrically on the chosen $\Lambda$. We iteratively adjust $\Lambda$ until $u(L)=-u_{L}$ to within a specified tolerance (usually $1 \%$ of $u_{L}$ ).

At this point, chemistry is turned on, and the computation is continued until full convergence is obtained. In this way, we start the reacting solution with an initial guess for the temperature profile that is very close to the correct one, since under these conditions chemistry has only a minor effect on $T(z)$. This significantly speeds up convergence, since the chemical production terms in the species equations are strongly temperature dependent.

We solve the equations on a fixed mesh, spaced uniformly in the variable $\eta(z)$, given by

$$
\eta / L=\left(1-e^{-a z / L}\right) /\left(1-e^{-a}\right),
$$

where $a$ is a fixed constant. The limit $a \rightarrow 0$ gives a uniform mesh, while $a>0$ concentrates more points near the substrate. For the cases discussed below with 25 species, 56 reactions, and a gas composition of $0.5 \%$ methane in hydrogen, typical run times are $2 \mathrm{~h}$ on a VAXstation 3100 workstation running VMS, using a relative error tolerance of $10^{-4}$. For higher initial methane concentrations, the run times can be several times longer. For the cases considered here, convergence was always obtainable using the damped Newton method alone, and therefore time integration was not necessary.

\section{SIMULATIONS OF THE FILAMENT-ASSISTED ENVIRONMENT}

Quantitative gas-phase concentrations have been measured during filament-assisted diamond growth by two groups: Harris et al. have measured stable species concentrations at the substrate using sampling mass spectroscopy, ${ }^{5-7}$ and Celii et $\mathrm{al}$. have reported concentrations for $\mathrm{CH}_{4}, \mathrm{C}_{2} \mathrm{H}_{2}$, $\mathrm{CH}_{3}$, and $\mathrm{C}_{2} \mathrm{H}_{4}$, measured with infrared diode laser absorption spectroscopy. ${ }^{2-4}$ To the best of our knowledge, these measurements are the only quantitative absolute concentrations reported to date for any diamond growth environment.

We have applied our model to simulate the experimental conditions of Harris and co-workers ${ }^{5}$ and of Celii et al. ${ }^{3}$ The simulation is necessarily approximate, since the geometry of neither experiment is one-dimensional. Both employ standard hot-filament configurations, with a single tungsten filament suspended 0.5 to $2 \mathrm{~cm}$ from the substrate. Three-dimensional effects can enter, for example, through diffusion of gas from the cold regions away from the filament to the substrate. This is evident in the results of Ref. 5 at large filament-substrate separations, for which the acetylene mole fraction decreases and the methane mole fraction approaches the initial, unreacted value. Since the destruction of acetylene under these conditions is very slow, this effect is presumably due to dilution of acetylene created near the filament with unreacted gas.

Another three-dimensional effect arises due to thermal diffusion, which can reduce the overall carbon content of the gas near the filament and substrate with respect to the colder wall regions by as much as a factor of $2 .^{6}$ Still another $3 D$ effect enters in interpreting the concentrations reported in Ref. 3, since these are averaged over the line of sight of the absorption measurements, which encompasses both hot and cold gas regions. For these reasons, we can only expect approximate agreement between our calculated concentrations and the measured values.

The actual chemistry occurring at the filament is not yet well understood. It is likely that both homogeneous and heterogenous chemistry contribute to $\mathrm{H}_{2}$ dissociation and methane conversion. The near-filament region also clearly deviates from our assumption of a one-dimensional geometry. Consequently, we do not attempt to model these nearfilament processes in detail. We simply take the gas composition and temperature at the gas inlet in our model problem to be characteristic of gas which has passed "near" the filament. Following Harris and co-workers, ${ }^{5}$ we estimate the near-filament gas composition by allowing the initial gas mixture to evolve isothermally at a specified temperature for a residence time $t_{\text {res }}$. Based on the gas temperature measurements of Ref. 5, we take this temperature to be $2000 \mathrm{~K}$.

We choose $t_{\text {res }}$ to yield approximately the 1:1 $\mathrm{CH}_{4} / \mathrm{C}_{2} \mathrm{H}_{2}$ ratio measured in Ref. 5 near the filament. Using the kinetic mechanism discussed below at $2000 \mathrm{~K}$, this 
occurs at about $t_{\mathrm{r} w}=0.2 \mathrm{~s}$ for a $0.29 \% \mathrm{CH}_{4} / \mathrm{H}_{2}$ mixture. For consistency, we use $2000 \mathrm{~K}$ and $0.2 \mathrm{~s}$ residence time to calculate the gas inlet composition for all cases we consider.

The primary effect of the assumed residence time is on the methane/acetylene ratio. Since at equilibrium at $2000 \mathrm{~K}$ most of the initial methane would be converted to acetylene, the measured 1:1 ratio shows that equilibrium is not attained near the filament. At $t_{\mathrm{res}}=0.2 \mathrm{~s}$, the atomic $\mathrm{H}$ concentration is within a factor of 2 of its equilibrium value for all cases we consider. As will be shown below, most minor hydrocarbons are strongly affected by chemistry between the filament and the substrate; consequently, their concentrations at the substrate are only weakly dependent on their assumed values at the filament (but may be more strongly affected by $t_{\text {res }}$ through dependence on $[\mathrm{H}],\left[\mathrm{CH}_{4}\right]$, and/or $\left[\mathrm{C}_{2} \mathrm{H}_{2}\right]$ ).

The kinetic mechanism is based on that used by Harris ${ }^{26}$ in previous simulations of filament-assisted diamond growth. For $C_{1}$ and $C_{2}$ chemistry, we use the mechanism given in Ref. 26 , although we include pressure-dependent rate constants for the unimolecular reactions describing pyrolysis of $\mathrm{CH}_{4}, \mathrm{C}_{2} \mathrm{H}_{6}, \mathrm{C}_{2} \mathrm{H}_{5}$, and $\mathrm{C}_{2} \mathrm{H}_{3}$ (reactions A2, A 14, $\mathrm{A} 15$, and A22, respectively, of Ref. 26). The limiting highpressure rate constants are taken from Warnatz ${ }^{27}$ for these reactions, and we assume a Lindemann falloff function. We include chemistry up through the $\mathrm{C}_{4}$ hydrocarbons, taking the relevant $C_{3}$ and $C_{4}$ reactions from $R$ ef. 28 . The complete model consists of $\mathrm{H}, \mathrm{H}_{2}$, and 23 hydrocarbons, with 56 reactions.

To model the experiments of Harris and co-workers, ${ }^{5}$ we take a gas composition of $0.29 \%$ methane in hydrogen at a pressure of 20 Torr. An inlet velocity of $1 \mathrm{~cm} / \mathrm{s}$ is assumed, and we take the substrate temperature to be $1000 \mathrm{~K}$. Since diamond growth rates are reported in Ref. 5 for a $0.5-\mathrm{cm}$ filament-substrate separation, we take $L=0.5 \mathrm{~cm}$.

Under these conditions, the Reynolds number $\operatorname{Re}=\rho u_{,} L / \mu$ based on inlet conditions is $5.3 \times 10^{4}$. The convective terms in Eqs. (2)-(7) are therefore negligible, and the velocity field is determined simply by viscous diffusion. Since the Prandtl number $\rho c_{p} \mu / k$ is of order one $(0.68)$, the temperature profile is also dominated by diffusion. and we expect approximately the linear heat-conduction profile. Species transport is also primarily by diffusion, since the largest mass-transfer Peclet number $u_{L} L / D$ has the value $2.3 \times 10^{-3}$. Consequently, the species profiles are largely decoupled from the velocity profiles, and independent of the assumed $u_{L}$ (verified numerically as well).

In a real hot-filament reactor, buoyancy-induced velocities will be present, which we estimate to be comparable to, or somewhat greater than, the convective velocity. However, the very small mass-transfer Peclet numbers indicate that even in the presence of buoyancy, mass transfer will be dominated by diffusion, since diffusion velocities will be roughly three orders of magnitude larger than buoyancy or convective velocities.

An approximate analytical solution describes the velocity and temperature fields for our model problem in the creeping flow limit $\mathrm{Re} \ll 1$. Assuming constant viscosity, the solution to Eq. (3) in the limit $\operatorname{Re} \ll 1$ is

$$
V(z)=-(\Lambda / 2 \mu) z(L-z) .
$$

For a general density profile $\rho(z)$, no analytical solution for $u(z)$ is possible. To obtain a simple analytical result, we approximate the density gradient term in Eq. (2) by a linear profile:

$$
\frac{u}{\rho} \frac{d \rho}{d z} \approx \frac{u_{L}}{L}\left(\frac{T_{i}-T_{s}}{T_{i}}\right) \frac{z}{L} .
$$

With this approximation, we find

$$
u(z)=u_{L}\left[2 \tilde{t}(z / L)^{3}-(2 \tilde{t}+1)(z / L)^{2}\right],
$$

where $\tilde{t}=\left(T_{i}+T_{\mathrm{s}}\right) / 2 T_{i}$. The radial pressure gradient eigenvalue is given approximately by

$$
\Lambda=-6 \mu u_{L} i / L^{3} .
$$

If the heat generation terms in the energy equation are negligible and the thermal conductivity is constant, then the temperature profile in this limit is simply

$$
T(z)=T_{s}+\left(T_{i}-T_{\mathrm{s}}\right)(z / L) .
$$

The velocity and temperature fields computed numerically for the conditions discussed above are shown in Fig. 1, along with the approximate results of Eqs. (12)-(16). It is apparent that the analytical expressions provide good estimates of the flowfield, even though variations in $\mu$ and $\lambda$ are actually non-negligible, and in spite of the approximate treatment of the density gradient term.

The mole fraction profiles calculated for these conditions are shown in Fig. 2 for all species reaching $0.1 \mathrm{ppm}$, assuming kinetically limited diamond growth (all sticking probabilities set to zero). The major species concentrations at the substrate are in qualitative agreement with the predictions of the constant-temperature kinetic analysis of Ref. 5 , although we find $\mathrm{C}_{2} \mathrm{H}_{4}$ and $\mathrm{C}_{2} \mathrm{H}_{6}$ concentrations about an order of magnitude lower than estimated in Ref. 5. We also find non-negligible concentrations at the substrate for $\mathrm{CH}_{2}$ and several $\mathrm{C}_{3}$ hydrocarbons. (Due to the uncertainties associated with $\mathrm{C}_{3}$ chemistry, the $\mathrm{C}_{3}$ profiles should be taken as indicative only.)

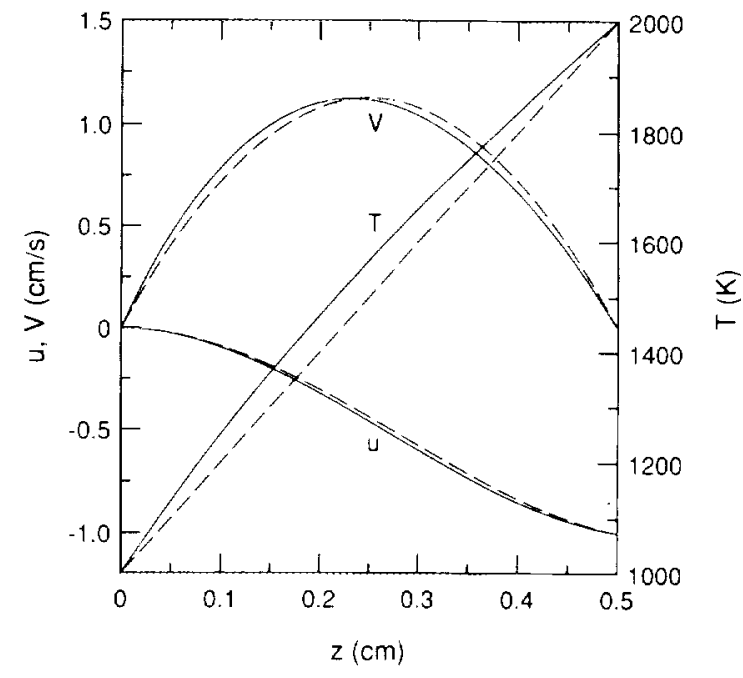

FIG. 1. Comparison of the exact velocity and temperature profiles calculated numerically (solid lines) with those calculated from the approximate analytical solution for the creeping flow limit (dashed lines). 


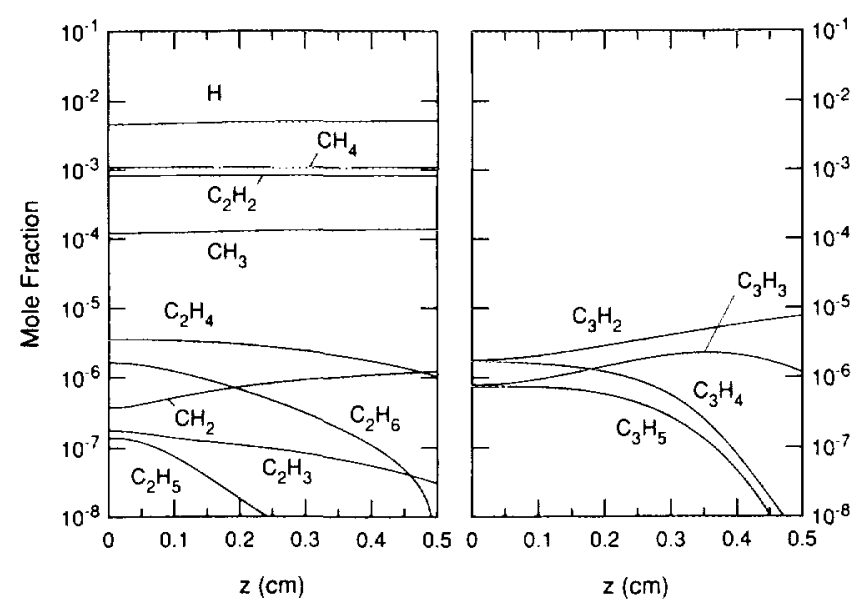

FIG. 2. Mole fraction profiles for all species reaching $0.1 \mathrm{ppm}$, calculated for conditions simulating experiments of Harris and co-workers (see Ref. 5).

Thermal diffusion has only a slight influence on the $\mathrm{H}$ atom concentration profile. From Eq. (9), the temperature gradient will induce an $\mathrm{H}$-atom concentration gradient given roughly by $\Delta X_{H} / X_{H} \approx \Theta_{H} \ln \left(T_{i} / T_{s}\right) / X_{H}$. For the present case, this gives $\Delta X_{H} / X_{H} \approx 10 \%$, which compares well with the actual calculated gradient.

Some insight into these concentration profiles may be gained by examining the relative effects of chemistry and diffusion. A local chemical timescale for each species is given by the chemical destruction time $\tau_{\text {chem }}(z)$, defined as the molar concentration divided by the molar destruction rate. Each molecule exists, on average, a time $\tau_{\text {chem }}$ before being consumed again by chemistry. During this time, an average molecule will diffuse a distance $l_{d}$ normal to the substrate, given approximately by

$$
l_{d} \approx \sqrt{D \tau_{\text {chem }}} .
$$

If $l_{d} \ll L$, then the concentration profile is determined primarily by local chemistry. In the opposite limit, $l_{d} \gg L$, molecules may freely diffuse over distances larger than $L$ without reacting, and thus chemistry is unimportant in determining the species profile.

Profiles of $l_{d}$ for several species are shown in Fig. 3. These results show that the species $\mathrm{H}_{2}, \mathrm{C}_{2} \mathrm{H}_{2}$, and, to a lesser extent, $\mathbf{H}$, may diffuse relatively unimpeded from the filament to the substrate. Methyl radicals, on the other hand, may diffuse only about $1 \mathrm{~mm}$ before they are consumed, primarily through the fast bimolecular reaction

$$
\mathrm{CH}_{3}+\mathrm{H}_{2} \rightleftharpoons \mathrm{CH}_{4}+\mathrm{H} \text {. }
$$

Therefore, methyl radicals reaching the substrate do not diffuse directly from the filament, but are created through $\mathrm{CH}_{4}+\mathrm{H} \rightarrow \mathrm{CH}_{3}+\mathrm{H}_{2}$ near the substrate.

The gas composition is far from thermodynamic equilibrium in this system. Most species have concentrations orders of magnitude different from the values they would have if homogeneous equilibrium prevailed at the local temperature. The nonequilibrium is maintained primarily by diffusion of reactive species with large $l_{d}$ (e.g., $\mathrm{H}$ atoms). (Of course, if we consider heterogeneous equilibrium, allowing

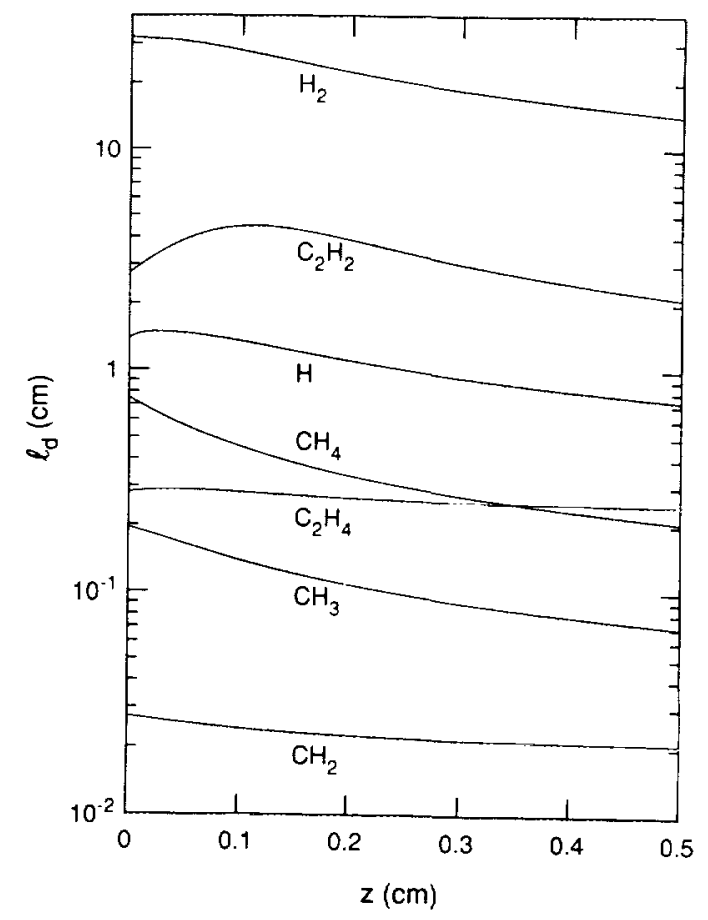

FIG. 3. Characteristic diffusion lengths for several species.

for precipitation of solid carbon, then all of the hydrocarbons are in superequilibrium, even if the gaseous species are in equilibrium with one another.)

However, several reactions are found to be nearly in partial equilibrium throughout the gas, including reaction (18). In particular, many (but not all) of the $\mathrm{H}$-abstraction reactions

$$
\mathrm{C}_{n} \mathrm{H}_{m}+\mathrm{H}_{2} \rightleftharpoons \mathrm{C}_{n} \mathrm{H}_{m+1}+\mathrm{H}
$$

are in partial equilibrium. A quantitative measure of partial equilibrium is provided by the partial equilibrium ratio $R_{\mathrm{eq}}$, defined for a general reaction of the form

$$
a A+b B \rightleftharpoons c C+d D
$$

$$
R_{\mathrm{eq}}=\frac{1}{K_{\mathrm{eq}}} \frac{[C]^{c}[D]^{d}}{[A]^{a}[B]^{b}} .
$$

For $R_{\text {eq }} \ll 1$ or $R_{\text {eq }} \gg 1$, the reaction proceeds irreversibly forward or backward, respectiveiy, while for $R_{\mathrm{eq}}=1$ the forward and backward rates are equal and partial equilibrium holds.

\section{The reactions}

$$
\begin{aligned}
& \mathrm{CH}_{2}+\mathrm{H} \rightleftharpoons \mathrm{CH}+\mathrm{H}_{2}, \\
& \mathrm{C}_{2} \mathrm{H}_{2}+\mathrm{H} \rightleftharpoons \mathrm{C}_{2} \mathrm{H}+\mathrm{H}_{2}, \\
& \mathrm{C}_{4} \mathrm{H}_{2}+\mathrm{H} \rightleftharpoons \mathrm{C}_{4} \mathrm{H}+\mathrm{H}_{2},
\end{aligned}
$$

are all highly equilibrated for $0 \leqslant x \leqslant L$, with $\left|1-R_{\text {eq }}\right|<7 \times 10^{-3}$. Although the radicals $\mathrm{C}$ and $\mathrm{C}_{2}$ are not included in our model, the analogous reactions $\mathrm{CH}+\mathrm{H} \rightleftharpoons \mathrm{C}+\mathrm{H}_{2}$ and $\mathrm{C}_{2} \mathrm{H}+\mathrm{H} \rightleftharpoons \mathrm{C}_{2}+\mathrm{H}_{2}$ would surely be in partial equilibrium as well under these conditions. Several other reactions are near partial equilibrium, as shown in Fig. 4. 


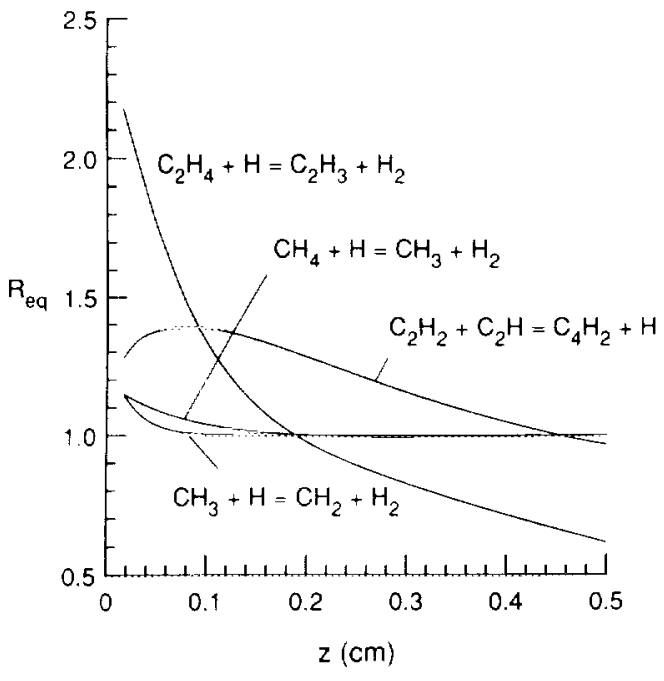

FIG. 4. Partial equilibrium ratios for several reactions.

The concentrations of all $\mathrm{C}_{1}$ radicals are therefore tightly linked to the local methane and $\mathrm{H}$-atom concentrations through relations of the form

$$
\left[\mathrm{CH}_{n}\right] \propto\left[\mathrm{CH}_{4}\right]\left(\frac{[\mathrm{H}]}{\left[\mathrm{H}_{2}\right]}\right)^{4 \cdot n}, \quad n=0-3,
$$

and $\mathrm{C}_{2}$ and $\mathrm{C}_{2} \mathrm{H}$ are similarly coupled to acetylene and $\mathrm{H}$ by

$$
\left[\mathrm{C}_{2} \mathrm{H}_{n}\right] \propto\left[\mathrm{C}_{2} \mathrm{H}_{2}\right]\left(\frac{[\mathrm{H}]}{\left[\mathrm{H}_{2}\right]}\right)^{2}, \quad n=0,1 .
$$

The proportionality factors are the (temperature-dependent ) equilibrium constants for the overall reactions implied by Eqs. (25) and (26).

We may ask which species are possible candidates as the diamond growth species. The growth rate is given in Ref. 5 as greater than $0.1 \mu \mathrm{m} / \mathrm{h}$; a subsequent estimate of the actual rate under these conditions is 0.5 to $1.0 \mu \mathrm{m} / \mathrm{h} .{ }^{29}$ Following the discussion given in Ref. 5, all of the species shown in Fig. 2 except $\mathrm{C}_{2} \mathrm{H}_{3}$ and $\mathrm{C}_{2} \mathrm{H}_{5}$ have surface collision rates high enough to account for this growt rate. However, a gasphase production rate large enough to maintain this surface flux is also required.

To investigate this question, we have carried out a set of runs for the above conditions, systematically allowing each minor hydrocabon species shown in Fig. 2 in turn to have a sticking probability of one, with all others zero. In this way, we may calculate upperbound, diffusion-limited growth rates for each assumed growth species. The growth rates so computed are given in Table I.

These results indicated that none of these species can account for the measured diamond growth rates. Thus, purely on grounds of gas-phase abundance the possible diamond growth species are limited to $\mathrm{CH}_{3}, \mathrm{C}_{2} \mathrm{H}_{2}$, and (possibly) $\mathrm{CH}_{4}$, though the low reactivity of $\mathrm{CH}_{4}$ would make it unlikely. If the methyl radical or acetylene is the primary growth species, then growth under these conditions is kinetically limited. For $\mathbf{C H}_{3}$, a sticking probability of $\gamma=4 \times 10^{3}$ gives a growth rate of $0.5 \mu \mathrm{m} / \mathrm{h}$, while for
TABLE I: Upper-bound, diffusion-limited diamond growth rates for various assumed growth species.

\begin{tabular}{cc}
\hline Species & Growth rate $(\mu \mathrm{m} / \mathrm{h})$ \\
\hline $\mathrm{CH}_{2}$ & 0.075 \\
$\mathrm{C}_{2} \mathrm{H}_{4}$ & 0.172 \\
$\mathrm{C}_{2} \mathrm{H}_{5}$ & 0.022 \\
$\mathrm{C}_{2} \mathrm{H}_{6}$ & 0.117 \\
$\mathrm{C}_{3} \mathrm{H}_{2}$ & 0.235 \\
$\mathrm{C}_{3} \mathrm{H}_{3}$ & 0.156 \\
$\mathrm{C}_{3} \mathrm{H}_{4}$ & 0.232 \\
$\mathrm{C}_{3} \mathrm{H}_{3}$ & 0.093 \\
\hline
\end{tabular}

$\mathrm{C}_{2} \mathrm{H}_{2}, \gamma=4 \times 10^{-4}$ would be required to produce this growth rate.

We next apply our model to simulate the experiments of Celii et al. ${ }^{2-4}$ Concentrations determined from absorption measurements are reported for a methane/hydrogen ratio of $f=1.0 \%, p=20$ Torr, and a filament temperature of $2200^{\circ} \mathrm{C}$. $^{3}$ To model this experiment, we use the same procedure as discussed above to determine the inlet gas composition, and we take a substrate temperature of $1200 \mathrm{~K}$ and $L=2 \mathrm{~cm}$.

Species profiles calculated for this case, with all sticking probabilities set to zero, are shown in Fig. 5. In comparison to the profiles discussed above, the $\mathrm{C}_{2} \mathrm{H}_{2} / \mathrm{CH}_{4}$ ratio is greater here, due to faster methane pyrolysis resulting from the higher initial methane concentration. For $f=1 \%$, the acetylene concentration attains $90 \%$ of its equilibrium value at the inlet after the assumed residence time of $0.2 \mathrm{~s}$, in contrast to $57 \%$ of equilibrium for $f=0.29 \%$. The $\mathrm{CH}_{4}$ and $\mathrm{CH}_{3}$ mole fractions are almost the same here as for the previous case.

Due to the larger inlet-substrate separation, $\mathrm{C}_{3}$ hydrocarbons now have sufficient time to be partially consumed before reaching the substrate, and therefore their concentrations at the substrate are lower. On the other hand, $\mathrm{C}_{4} \mathrm{H}_{2}$ and $\mathrm{C}_{4} \mathrm{H}$ now appear at the 1-ppm level. The $\mathrm{C}_{4} \mathrm{H}_{2}$ concentration is determined by the partial equilibrium of

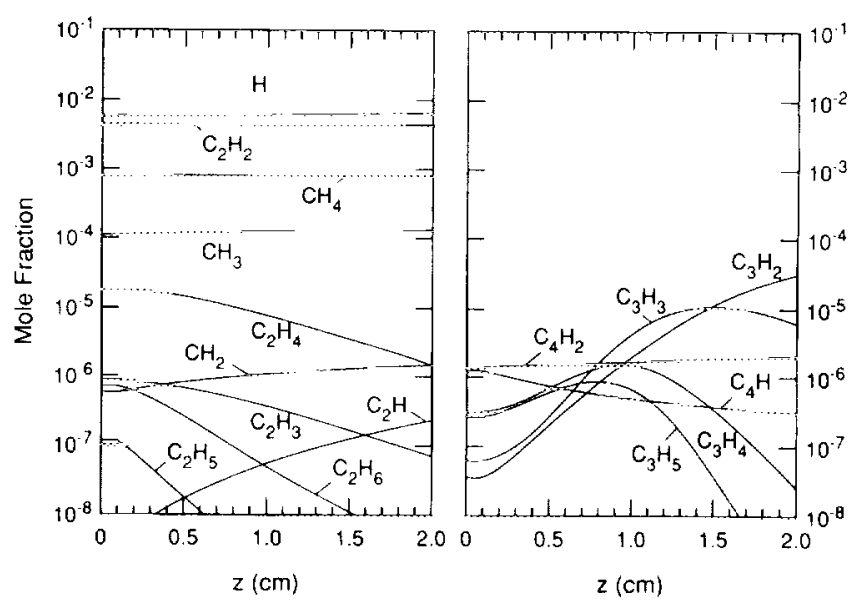

FIG. 5. Mole fraction profiles for all species reaching $0.1 \mathrm{ppm}$, calculated for conditions simulating experiments of Celii et al. (see Ref. 3 ).

D. G. Goodwin and G. G. Gavillet 
$\mathrm{C}_{2} \mathrm{H}_{2}+\mathrm{C}_{2} \mathrm{H} \rightleftharpoons \mathrm{C}_{4} \mathrm{H}_{2}+\mathrm{H}$ and $\mathrm{C}_{4} \mathrm{H}$ is maintained by $\mathrm{C}_{4} \mathrm{H}+\mathrm{H}_{2} \rightleftharpoons \mathrm{C}_{4} \mathrm{H}_{2}+\mathrm{H}$. Consequently, we expect

$$
\left[\mathrm{C}_{4} \mathrm{H}_{2}\right] \propto \frac{\left[\mathrm{C}_{2} \mathrm{H}_{2}\right]^{2}}{\left[\mathrm{H}_{2}\right]}
$$

and

$$
\left[\mathrm{C}_{4} \mathrm{H}\right] \propto\left[\mathrm{C}_{4} \mathrm{H}_{2}\right] \frac{[\mathrm{H}]}{\left[\mathrm{H}_{2}\right]} .
$$

We have not considered hydrocarbons larger than $\mathrm{C}_{4}$ species in our model; similar partial equilibria may occur for other, heavier hydrocarbons. Although these species are probably insignificant as growth species, aromatic hydrocarbons (not included in our model) have been postulated to contribute to graphitic $s p^{2}$ carbon deposition. ${ }^{15}$ This sug. gests that modeling heavier hydrocarbons may be important to predict $s p^{2} / s p^{3}$ ratios.

The number densities reported by Celii et al.,$^{3}$ measured with an optical path passing $0.5 \mathrm{~cm}$ from the substrate, are compared in Table II with the values we calculate at $z=0.5$ $\mathrm{cm}$. For most species, we find reasonably good agreement. Our results confirm the failure to detect $\mathrm{C}_{3} \mathrm{H}_{4}, \mathrm{C}_{3} \mathrm{H}_{6}$, and $\mathrm{C}_{4} \mathrm{H}_{2}$ above the detection limit of the ir absorption measurements. The largest discrepancies are seen in the measured and calculated $\mathrm{CH}_{4}$ and $\mathrm{CH}_{3}$ concentrations. The most likely factor responsible for the discrepancy is the line-of-sight nature of the diode laser measurements, ${ }^{3}$ which were made with a pathlength of $25 \mathrm{~cm}$ within the chamber. The nonnegligible contribution from gas in cold regions far from the filament or substrate is indicated by the measured acetylene rotational temperature of $200-300 \mathrm{~K}$

If it is assumed that $\mathrm{CH}_{3}$ is primarily localized along the filament $(3 \mathrm{~cm}$ in length), then the local methyl concentration would be 10 times larger than the pathlength-averaged value, and would agree very well with our calculated methyl number density. The discrepancies for $\mathrm{CH}_{4}$ and $\mathrm{C}_{2} \mathrm{H}_{2}$ can similarly be understood, since cold regions would be expected to contain significant amounts of unreacted methane, and correspondingly less acetylene than in the hot region. In light of these considerations, the agreement between calculated and measured values is probably as good as can be expected for a one-dimensional model.

Butler and Celii ${ }^{4}$ have also reported the interesting result that the spatially resolved atomic H REMPI (resonance-enhanced multiphoton ionization) signal decreases

TABLE II: Comparison of measured ${ }^{\mathrm{a}}$ number densities with those calculated at $z=0.5 \mathrm{~cm}$

\begin{tabular}{lll}
\hline \hline Species & Measured $\left(\mathrm{cm}^{3}\right)$ & Calculated $\left(\mathrm{cm}^{3}\right)$ \\
\hline $\mathrm{CH}_{4}$ & $8 \pm 3 \times 10^{14}$ & $1.1 \times 10^{14}$ \\
$\mathrm{CH}_{3}$ & $2 \pm 1.5 \times 10^{12}$ & $1.6 \times 10^{13}$ \\
$\mathrm{C}_{2} \mathrm{H}_{2}$ & $2 \pm 1 \times 10^{14}$ & $6.0 \times 10^{14}$ \\
$\mathrm{C}_{2} \mathrm{H}_{4}$ & $6 \pm 2 \times 10^{12}$ & $2.0 \times 10^{12}$ \\
$\mathrm{C}_{3} \mathrm{H}_{4}$ & $<8 \times 10^{13}$ & $9.4 \times 10^{10}$ \\
$\mathrm{C}_{3} \mathrm{H}_{6}$ & $<1.4 \times 10^{14}$ & $2.2 \times 10^{9}$ \\
$\mathrm{C}_{4} \mathrm{H}_{2}$ & $<3 \times 10^{13}$ & $2.0 \times 10^{11}$ \\
\hline
\end{tabular}

Reference 3 .

"Sum of methyl acetylene and cyclopropane. by an order of magnitude as $f$ increases from zero to $3 \%$. As they point out, this cannot be explained on the grounds of equilibrium thermodynamics. Two possibilities exist: If heterogeneous chemistry is responsible for $\mathrm{H}$-atom production at the filament, then a variation in the catalytic efficiency with gas composition due to hydrocarbon poisoning of the filament surface could be responsible. A second possibility is that enhanced gas-phase recombination of $\mathrm{H}$ at higher $f$ could lead to a lower $\mathrm{H}$-atom concentration where the REMPI measurements were made. ${ }^{30}$ We cannot answer questions about heterogenous chemistry with our present model, but we may examine the possibilities for homogeneous recombination of $\mathrm{H}$ atoms.

In the hot-filament environment at low $f, \mathrm{H}$-atom recombination is slow, occurring primarily through

$$
\mathrm{H}+\mathrm{C}_{2} \mathrm{H}_{2}+\mathrm{M} \rightarrow \mathrm{C}_{2} \mathrm{H}_{3}+\mathrm{M} \text {, }
$$$$
\mathrm{C}_{2} \mathrm{H}_{3}+\mathrm{H} \rightarrow \mathrm{C}_{2} \mathrm{H}_{2}+\mathrm{H}_{2} \text {, }
$$

and

$$
\begin{aligned}
& \mathrm{H}+\mathrm{CH}_{3}+\mathrm{M} \rightarrow \mathrm{CH}_{4}+\mathrm{M}, \\
& \mathrm{CH}_{4}+\mathrm{H} \rightarrow \mathrm{CH}_{3}+\mathrm{H}_{2} .
\end{aligned}
$$

A variety of other recombination paths involving $\mathrm{C}_{3}$ and $\mathrm{C}_{4}$ hydrocarbons become increasingly important as $f$ increases. The total recombination rate through hydrocarbon channels is much larger than the direct recombination rate due to $\mathrm{H}+\mathrm{H}+\mathrm{M} \rightarrow \mathrm{H}_{2}+\mathrm{M}$ for typical hot-filament conditions. A characteristic recombination time may be defined as

$$
\tau_{\text {rec }}=[\mathrm{H}] /\left|\dot{\omega}_{\mathrm{H}}\right| \text {, }
$$

where $\left|\dot{\omega}_{\mathbf{H}}\right|$ is the net chemical disappearance rate of $\mathbf{H}$ atoms. [Note that $\tau_{\text {rec }} \gg \tau_{\text {chem }}$, since the net consumption of $\mathrm{H}$ is rate-limited by the slow three-body steps of Eqs. (29a) and (30a).] Profiles of $\tau_{\text {rec }}(z)$ are shown in Fig. 6 for $f=0.5 \%$ and $1.0 \%$. For comparison purposes, the recombination time for the direct process $\mathrm{H}+\mathrm{H}+\mathrm{M} \rightarrow \mathrm{H}_{2}+\mathrm{M}$ is also shown, calculated for the conditions of the $f=0.5 \%$ case.

The quantity $\tau_{\text {rec }}^{-1}$ is the probability per unit time of an $\mathrm{H}$

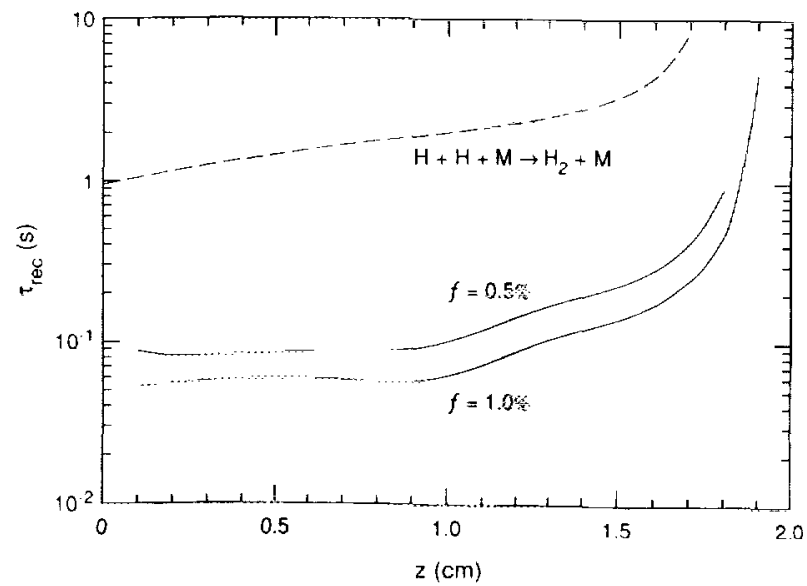

FIG. 6. H-atom recombination times for $f=0.5 \%$ and $1.0 \%$. The dashed curve shows the recombination time for the direct reaction $\mathbf{H}+\mathbf{H}+\mathbf{M} \rightarrow \mathrm{H}_{2}+\mathbf{M}$, calculated for the conditions of the $f=0.5 \%$ case. 
atom irreversibly recombining. Making the reasonable assumption that the average recombination cross section is not strongly dependent on gas composition, this quantity must be proportional to the collision frequency of an $\mathrm{H}$ atom with all hydrocarbons. Since the total hydrocarbon concentration scales at most linearly with $f, \tau_{\text {rec }}{ }^{\prime}$ cannot increase faster than $f$. The results shown in Fig. 6 verify this reasoning, since $\tau_{\text {rec }}$ differs by somewhat less than a factor of 2 between the cases $f=0.5 \%$ and $1.0 \%$.

Consequently, at $f=3 \%, \tau_{\text {rec }}$ must be at least $20 \mathrm{~ms}$. Converting this time to a diffusion length $l_{\text {rec }}=\sqrt{D} \tau_{\text {rec }}$, we find that $l_{\text {rec }}$ will be at least $4.5 \mathrm{~cm}$ at $f=3 \%$. We conclude, then, that homogeneous recombination of $\mathrm{H}$ will only significantly affect the $\mathrm{H}$ concentration profile beyond $4 \mathrm{~cm}$ from the filament. Since the REMPI $\mathrm{H}$-atom measurements were made $0.8 \mathrm{~cm}$ from the filament, these results suggest strongly that the order-of-magnitude decrease in $[\mathrm{H}]$ observed in Ref. 4 as $f$ is increased to $3 \%$ cannot be explained on the basis of homogeneous recombination. The most likely explanation, then, is heterogeneous chemistry at the filament surface. This in turn implies that heterogeneous, rather than homogeneous, chemistry must be primarily responsible for $\mathbf{H}$-atom production under typical hot-filament conditions.

\section{SUMMARY}

In this paper, we have presented a one-dimensional model for simulation of the gaseous diamond growth environment, which allows calculation of velocity, temperature, and species concentration profiles. We have applied the model to simulate the hot-filament experiments of Harris and co-workers ${ }^{5}$ and Celii et al. ${ }^{3}$ To our knowledge, this represents the first detailed simulation of the filament-assisted diamond growth environment.

Calculations of upper-bound growth rates for various assumed growth species indicate that no hydrocarbon species other than $\mathrm{CH}_{3}, \mathrm{C}_{2} \mathrm{H}_{2}$, or $\mathrm{CH}_{4}$ is present in sufficient quantities to account for the meastired growth rate under these conditions. We also find that although the gas composition is far from equilibrium, a number of reactions are very close to partial equilibrium. In particular, the concentrations of all $\mathrm{C}_{1}$ hydrocarbon radicals are determined by the local $\mathrm{CH}_{4}$ and $\mathrm{H}$ concentrations, through partial equilibria of bimolecular $\mathrm{H}$-abstraction reactions. Similarly, the concentrations of $\mathrm{C}_{2}$ and $\mathrm{C}_{2} \mathrm{H}$ are determined only by the local $\mathrm{C}_{2} \mathrm{H}_{2}$ and $\mathrm{H}$ concentrations.

We calculate species concentrations in reasonable agreement with the values measured using infrared diode laser absorption spectroscopy. The largest discrepancies may be attributed to the pathlength-averaged nature of the measurements. ${ }^{3}$ We have also examined homogeneous $\mathrm{H}$ atom recombination in this environment, and find the rate insufficient to explain the large decrease in the $\mathrm{H}$ concentration observed by Butler and Celii ${ }^{4}$ when the methane con- centration is increased. These results suggest that heterogeneous chemistry at the filament is primarily responsible for $\mathrm{H}$-atom production.

\section{ACKNOWLEDGMENT}

This work is supported in part by the Office of Naval Research, Contract No. N00014-90-J-1386.

' J. C. Angus, F. A. Buck, M. Sunkara, T. F. Groth, C. C. Hayman, and R. Gat, Mater. Res. Bull. 14, 38 (1989).

${ }^{2}$ F. G. Celii, P. E. Pehrsson, H.-T. Wang, and J. E. Butler, Appl. Phys Lett. 52, 2043 (1988).

'F. G. Celii, P. E. Pehrsson, H.-T. Wang, H. H. Nelson, and J. E. Butler, Proceedings of the First International Conference on New Diamond Science and Technology, Tokyo, 1988 (in press).

${ }^{4} \mathrm{~J}$. E. Butler and F. G. Celii, Proceedings of the First International Conference on Diamond and Diamond-Like Films, edited by J. P. Dismuke (The Electrochemical Society, Pennington, NJ, 1989), pp. 317-324. 'S. J. Harris, A. M. Weiner, and T. A. Perry, Appl. Phys. Lett. 53, 1605 (1988).

${ }^{6}$ S. J. Harris, D. N. Belton, A. M. Weiner, and S. J. Schmieg, J. Appl. Phys 66, 5353 (1989).

${ }^{7}$ S. J. Harris and A. M. Weiner, Appl. Phys. Lett. 55, 2179 (1989)

${ }^{*}$ M. Frenklach and K. E. Spear, J. Mater. Res. 3, 133 (1988)

"M. Tsuda, M. Nakajima, and S. Oikawa, J. Am. Chem. Soc. 108. 5780 (1986).

10 S. J. Harris, Appl. Phys. Lett. 56, 2298 (1990).

"S. M. Valone, MRS Symp. Proc. 155, 227 (1989).

${ }^{12}$ L. R. Martin and M. W. Hill, J. Mater. Sci. Lett. 9, 621 (1990).

${ }^{13}$ W. G. Breiland, P. Ho, M. E. Coltrin, R. J. Kee, and G. H. Evans, MRS Symp. Proc. 117, 23 (1988)

${ }^{14}$ D. G. Goodwin, in Technology Update on Diamond Films, edited by R. P H. Chang, D. Nelson, and A. Hiraki (Materials Research Society, Pittsburgh, PA, 1989) pp. 153-156.

${ }^{15}$ M. Frenklach, J. Appl. Phys. 65, 5142 (1989)

${ }^{16} \mathrm{M}$. Frenklach, SDIO-IST/ONR Diamond Technology Initiative Symposium, 1989, paper T10

'M. E. Coltrin. R. J. Kee, and G. H. Evans, J. Electrochem. Soc. 136, 819 (1989).

${ }^{18}$ G. Evans and R. Greif, J. Heat Transfer 109, 928 (1987)

${ }^{14}$ R. B. Bird, W. E. Stewart, and E. N. Lightfoot, Transport Phenomeno (Wiley, New York, 1960).

${ }^{211}$ R. J. Kee, F. M. Rumpley, and J. A. Miller, Sandia National Laboratories Report SAND89-8009 (1989).

R. J. Kee, J. Warnatz, and J. A. Miller, Sandia National Laboratories Report SAND83-8209 (1983).

M. E. Coltrin, R. J. Kee, and J. A. Miller, J. Electrochem. Soc. 133, 1206 (1986).

"S. A. Self (unpublished)

${ }^{24} \mathrm{H}$. Motz and H. Wise. J. Chem. Phys. 31, 1893 (1960)

$\because$ J. F. Grcar, R. J. Kee, M. D. Smooke, and J. A. Miller, Twenty-first Symposium (International) on Combustion (The Combustion Institute, Pittsburgh, PA, 1986), pp. 1773-1782

${ }^{2}$ S. J. Harris, J. Appl. Phys. 65, 3044 ( 1989)

$27 \mathrm{~J}$. Warnatz, in Combustion Chemistry, edited by W. C. Gardiner, Jr. (Springer, New York, 1984), pp. 197-360.

${ }^{2 *}$ S. J. Harris, A. M. Weiner, and R. J. Blint, Combust. Flame 72, 91 (1988).

2"S. J. Harris (private communication)

"'W. A. Yarbrough and R. Messier, Science 247, 688 (1990). 